\title{
BIM4LIFE: GNSS AND BIM DATA FUSION FOR MAPPING HUMAN-MACHINE INTERACTION
}

\author{
Olga Golovina ${ }^{1}$ and Jochen Teizer ${ }^{2}$
}

\begin{abstract}
This paper presents an effective approach towards integrating Real-time Location Sensing (RTLS) and Building Information Modeling (BIM) data for mapping near hit events related to human-machine interaction on construction sites. The study - under the concept called BIM for lean and injury free environments (BIM4LIFE) focuses on key managerial and technological issues in planning safe and productive work environments: (a) the reliance of current performance measurement practices on lagging instead of adapting to leading indicator data and (b) the common unstructured nature and dynamic progress of construction work environments making it difficult to collect data that leads to quality information. Both call for reliable information and communication technology (ICT) in infrastructure and information management processes to advance safety in construction. The data employed are trajectories from Global Navigation Satellite System (GNSS) data loggers, while an as-is building information model and a-priori collected true geometric equipment information are the other main data sources. The initial result of the data fusion process is a heat map that precisely analyses pedestrian worker and equipment interactions. The novelty of this work lies in solving the interface issues from RTLS data to BIM and to automated protective safety equipment modelling. The methods were tested in realistic work settings. The paper concludes with a critical review on the reliability of the methods employed as well as an outlook on possible changes to current work practices.
\end{abstract}

Keywords: BIM4LIFE, construction safety, remote sensing, equipment operator visibility, human-machine interaction, building information modelling (BIM)

\section{INTRODUCTION}

Construction safety performance is often measured through lagging indicators such as counting numbers of illnesses, injuries, and fatalities. Acting upon active leading indicators, such as capturing and analyzing proximity events between pedestrian workers and equipment, provide an additional metric, but it is hardly ever performed in practice. Once available though in large depth, it can assist a construction project team in their task, for example, for identifying and assessing human-machine interactions and safe guarding the detected hazardous work areas in or near real-time.

This paper presents a novel approach for recording, identifying, and analyzing interactive hazardous close call situations between pedestrian workers and heavy construction equipment. Spatiotemporal real-time location sensing (RTLS) data are analyzed to automatically measure a hazard index that is visualized in form of a heat map in an as-is building information model. The graphical representation of computationally identified worker-individual performance values in up-to-date building information

1 Ph.D. candidate, Chair of Computing in Civil Engineering, Ruhr-University Bochum, Germany, olga.golovina@rub.de.

2 Ph.D., Dipl.-Ing., Chair of Computing in Civil Engineering, Ruhr-University Bochum, Germany, jochen.teizer@rub.de. 
models allows automatically-generated personalized safety reports. This work therefore contributes new attributes for identifying and modeling risk, for example, identifying near miss locations while considering the actual conditions and progress of the work environment. Validating the root causes that lead to visibility-related incidents, in particular as they relate to potentially poor visibility of equipment operators, is important in preventing accidents on construction sites in the first place. The work thereby possibly transforms the field of practice in prevention through design $(\mathrm{PtD})$ and safety education and training concepts.

\section{BACKGROUND}

\subsection{Construction Industry Safety Records}

Approximately 60,000 construction workplace deaths occur worldwide every year causing loss of many lives, severe tragic consequences to many families, and significant collateral damages. Hinze and Teizer (2011) reported that $100 \%$ of all construction workers will experience an injury in their work life, while equipment is involved in about $25 \%$ of all fatal cases. Latter can be explained by limited situational awareness of humans or poor to no visibility of either pedestrian worker or equipment operator. They further found that up to $87 \%$ of all visibility-related construction equipment fatalities were absolutely not predictable by either operator or pedestrian worker. Other data, for example reported in an internal audit of a large insurance company, mentioned the monetary losses related to fatal construction equipment accidents to be 11.2 billion USD in between the years 2004 to 2006 (Waehrer et al. 2007). These high numbers are alarming and ask for change. One way out is the utilization of information and communication technologies (ICT) in construction safety.

\subsection{Accident Prevention Practices}

Construction industry leaders in safety have been focusing for a long time on "zero accidents"-techniques and other safety best practices to change organizational culture, supervision, preconditions, and unsafe personal behaviour (Teizer 2016). These industry leaders recognize now that their safety best practices are not enough to further decrease the numbers of accidents. It seems existing approaches have made their impact. Meanwhile, internet of things (IoT) and other technological innovations seem to rapidly transform the conduct of the construction businesses. The challenge though in construction is to transform the existing approaches towards adopting intelligent information and communication technologies (ICT). This comes to an industry that is known to be highly fragmented and conservative in applying innovation.

\subsection{Prevention through Design (PtD) and Construction Planning}

BIM is taking on a greater role in the industry, moving beyond clash detection and coordination to a valued resource in the field for lean jobsite planning Sacks et al. (2010). Research by Melzner et al. (2013) and Zhang, S. et al. (2015) have already shown concepts for intelligent and safe job site task planning, automated hazard identification, and risk prevention. Their approaches were implemented in some cases during design, but mostly during the construction planning stage. Examples of their pioneering work are Job Hazard Analysis (JHA) for on-site construction safety planning (see Figure 1a), 4D work packaging and task sequence planning (see Figure $1 \mathrm{~b}$ ), rule-checking to eliminate fall risk 
(see Figure 1c), and detection of unhealthy and ergonomically unsafe equipment installation positions at the design stage (see Figure 1d).

(a)

(c)
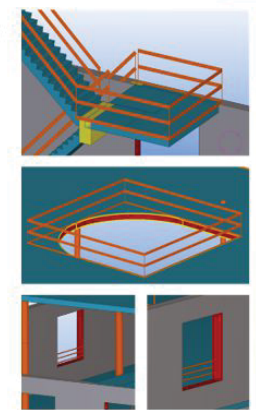
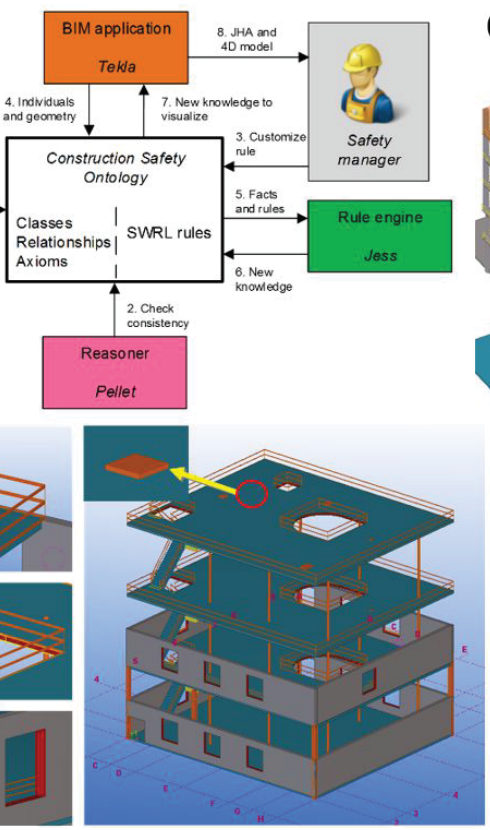

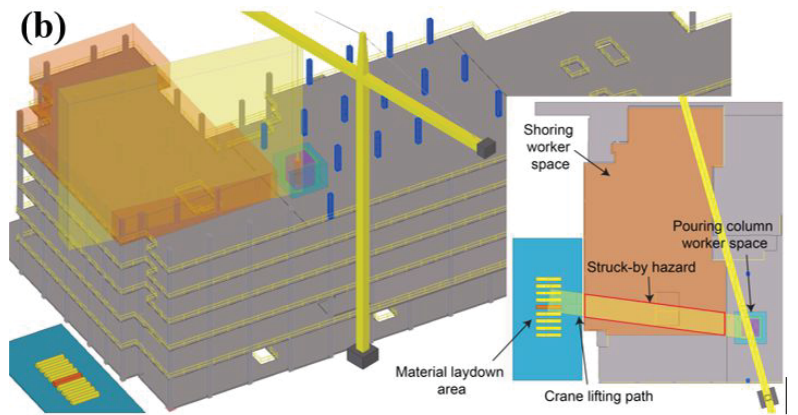

(d)

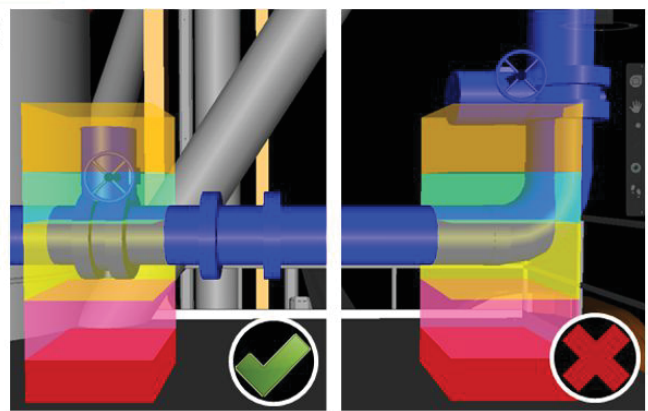

Figure 1: (a) Job Hazard Analysis (JHA) in BIM makes safety knowledge available for experts' decision making, (b) 4D work packaging and site sequence planning detects load

paths over pedestrian work crews, (c) fall hazard detection and protection using automatic rule checking in BIM, and (d) detecting valve heights out of reach for humans early in the design prevents long term illnesses

\section{METHODS AND SCOPE}

The study used a variety of mobile sensing equipment to gather the required data for mapping near hits in BIM. Siebert and Teizer (2014) used an unmanned aerial vehicle (UAV) to collect as-is information (a georeferenced 3D point cloud, see Figure 2, middle image) of a construction site (the same site explored in this study). Although a point cloud data set allows easily the visualization of existing workspace conditions from above ground (see Figure 2, left image), the data set was also used for manual progress capturing of the cast-in place concrete elements in the basement floor of the building under construction (see Figure 3, right). Although some early research methods exist to link point clouds to BIM (e.g., Bosche et al. 2008, Tang et al. 2010, Zhang, G. et al. 2015), the manual effort to detect every object from a raw point cloud is still high and may, according to the existing results (i.e., errors) and at least for practitioners, not warrant the investment in automatic point cloud to as-is BIM models quite yet.
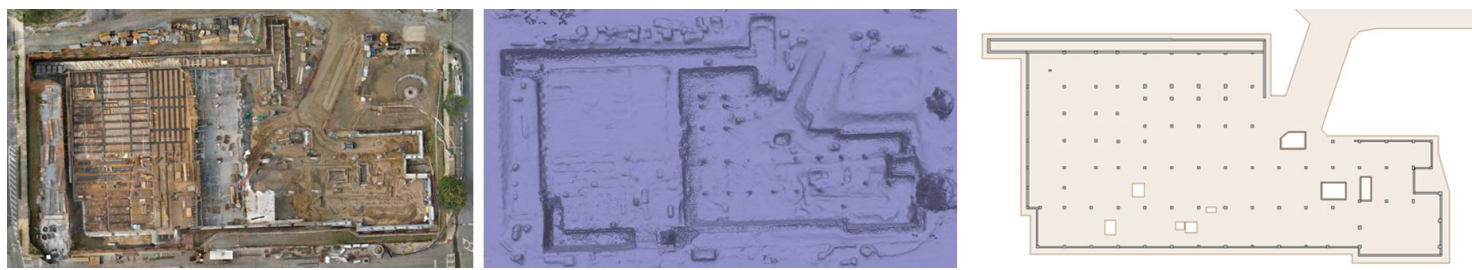

Figure 2: UAV-based photogrammetry for georeferenced point cloud generation and asis extraction of geometric building model information (from left to right) 
Though a fair amount of efforts are underway in that part of international research, the next step in this research study focused on collecting accurate geometric information of equipment used on construction sites. The specific application that was investigated (see later in the experimental part of the paper) focused on a skid steer loader hauling gravel on a busy construction site. This type of equipment can be very fast and mobile (i.e., rotate quickly) and thus exposes very high dangers to pedestrian workers getting hit. Earlier research by Teizer et al. (2010) measured accurate blind spot diagrams using laser scanning (see Figure 3). This study added the conversion of equipment blind spot diagrams to its own objects in BIM (see Figure 3, right) (Golovina et al. 2016). By doing so, it allowed, in a next step, employing a ray-tracing algorithm similar to Ray and Teizer (2016) to measure the size of the blind spots caused by the equipment in real-time. It further allowed visualizing the blind spots in BIM along with the trajectory information collected by commercially-available Global Navigation Satellite System (GNSS) data loggers that were attached to pedestrian workers and equipment before the GNSS data collection had started on the site (see Figure 4) (Pradhananga and Teizer 2013; Pradhananga 2014).
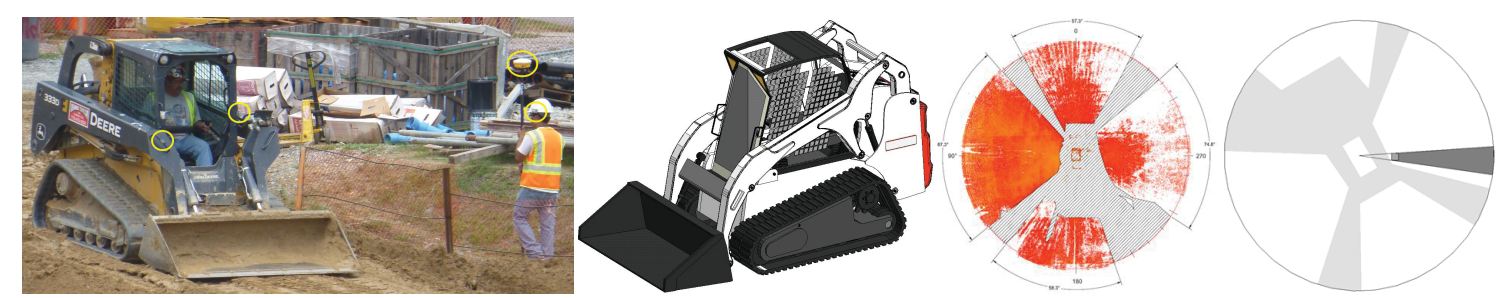

Figure 3: GNSS data evaluation using high-precision differential GPS unit, geometric equipment model (skid steer loader), blind spot diagram of the equipment as used in the field on the $12 \mathrm{~m}$ visibility blind spot measurement test circle, and geometric blind spot model encountering an obstacle (cast-in place concrete column) in BIM and causing an obstruction to the field-of-view of the equipment operator (from left to right)

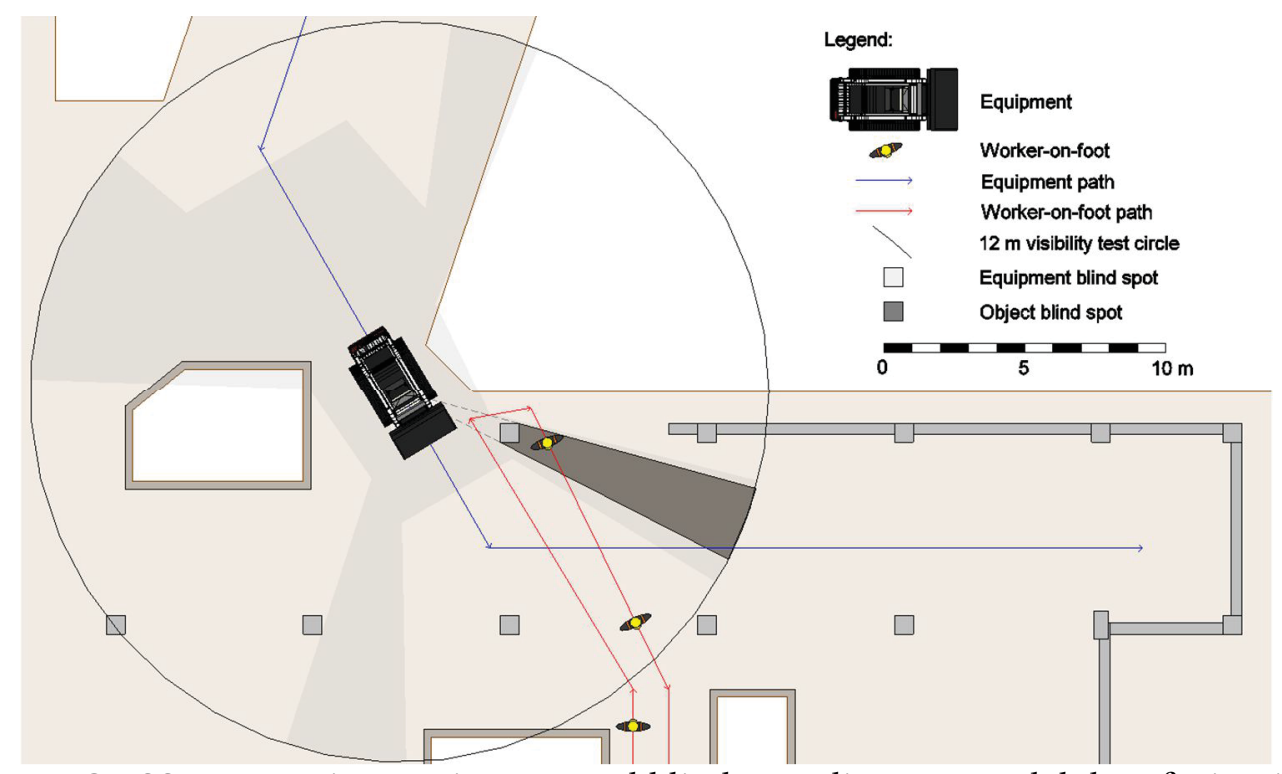

Figure 4: GNSS trajectories, equipment and blind spot diagram model data fusion in the as-is building information model 


\section{EXPERIMENT AND PRELIMINARY RESULTS}

The developed method for recording, identifying and analysing - previously undetected interactive hazardous near-miss situations between pedestrian workers and heavy equipment was tested at a building under construction. Using the spatiotemporal GNSS data from pedestrian workers and equipment to automatically measure a hazard index on a heat map allows a project team to automatically generate a site safety performance report and even personalized safety performance reports (see Figure 5). These reports, containing in this study mostly the risk exposure to equipment, will help define and verify geometric safety parameters, such as the entry of pedestrian workers in equipment blind spots. It also enables practitioners in determining the root causes that lead to equipmentand visibility-related incidents on construction sites, thereby transforming the field of practice in safety training and prevention.

In the specific application, the project safety manager identified a significant number of near hit events between several pedestrian workers and the equipment during the material hauling operation on the ramp into the excavated pit (see Figure 5, left image). Based on the findings and visual interpreting, the safety manager concluded to install a second entry/exit into the pit that was required by law anyway (see Figure 5, right image).

The information provided by the developed approach suggested an additional safety enhancement, the installing of red safety barricades (as modelled in BIM and shown in Figures 6 and 7) that keep the pedestrian workforce out of any of the equipment pathways. Although it would have safe-guarded the temporary task of filling gravel material in a confined work area near the entry in the excavated pit, the suggestion was not carried out due to concerns of productivity losses to other equipment operations.
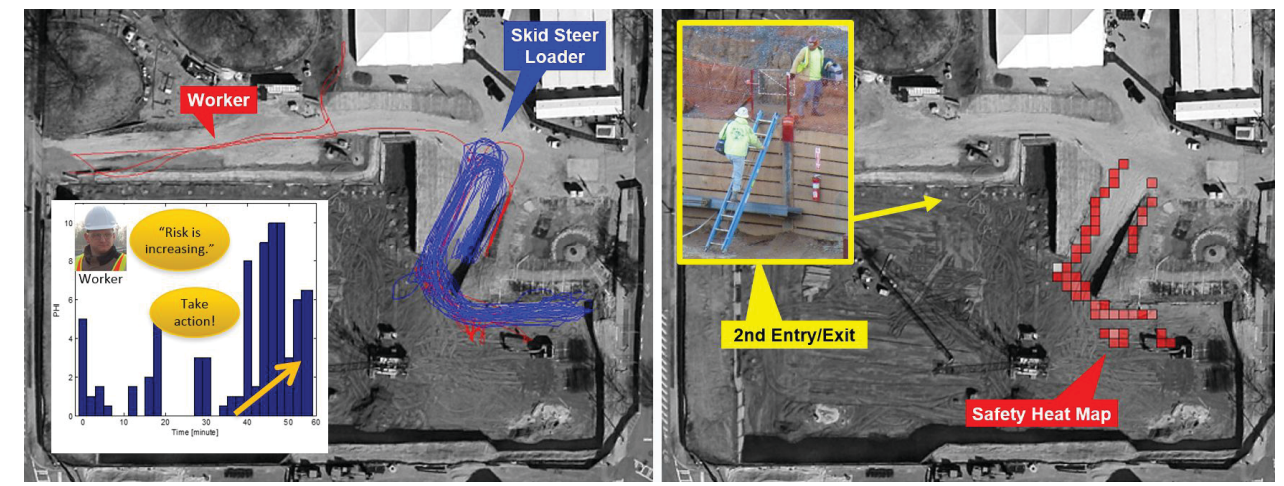

Figure 5: Trajectory data overlaid on a UAV image (left) and Safety Heat Map (occupancy grid) (right)

Field-realistic testing and interviews with the volunteering management and workforce were carried out to evaluate the acceptance of the technologies at the work stations and during the working hours.

The recorded site data can further be mined with existing statistical techniques and once new information for each individual resource has been created, additional personalized education and training (Teizer and Cheng 2015) can be issued, e.g. using the frequency of near hit events that can be quantified and mapped. If needed, a worker or equipment operator can be taught to change their individual working behavior - only if it is finally determined that at-risk behavior might lead to an accident. Alternatively, a piece of equipment that causes many near hits - once is has been observed probably for a longer time - can be replaced with a safer one. 


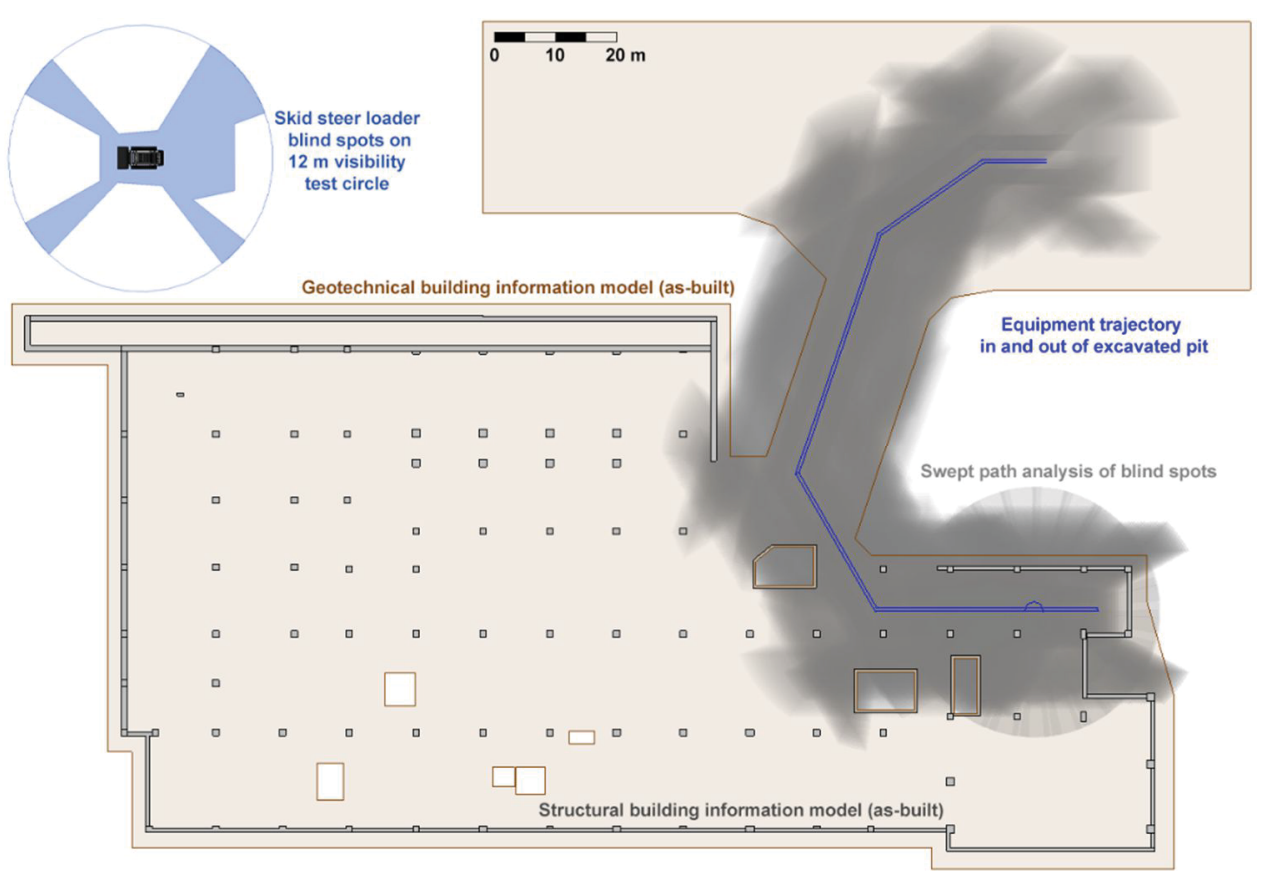

Figure 6: Swept path analysis: Invisible areas (mapped on the as-is BIM) due to equipment blind spots along the equipment travel path require additional protection

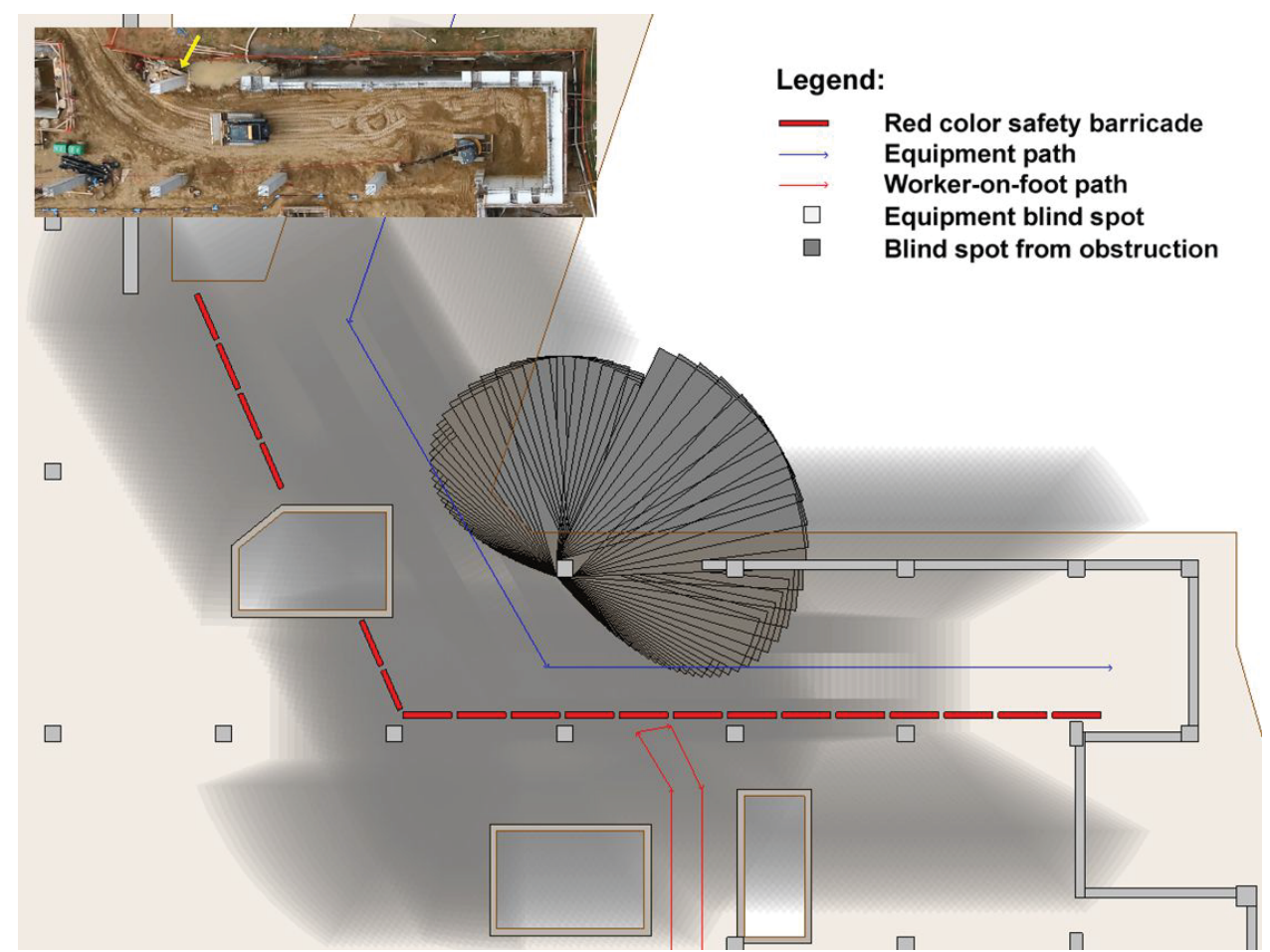

Figure 7: Modifying the as-is BIM by implementing safety objects that guard an active work area from pedestrian entry

Criteria on when to change will be set on leading indicators. The advantage of leading indicators (compared to lagging indicators) is that it can change the outcome of a process before it actually ends. The chance of improving the safety performance is subsequently high, as many incidents can be prevented if actions are taken rapidly enough. Thus, should 
an a-priori safety thresholds or tolerable benchmarks be met, control or actions will be taken to resolve the issue immediately, if not automatically. Such a scenario is novel as in the past, lagging indicators depended on incidents to occur to control a process after the fact. Lagging indicators also offered no remedy since information was provided too late to have significant impact on changing the process (and safety performance). As important site safety metrics become measurable effortless and at low investment, immediate increase in safety performance while a process is running, becomes possible.

\section{CONCLUSIONS}

Safety planning and monitoring in increasingly complex construction projects is challenging, because it is time consuming and still mostly a manual task for engineers and construction safety professionals. In such projects, timely communication of key essential safety information among the relevant stakeholders becomes essential to save lives and cost. When technology like GNSS data loggers, laser scanners for blind spot measurement, building information models, and smart computational algorithms to detect previously unidentified hazards (less obvious to humans) are utilized together to rapidly provide valuable information to decision makers, significant benefits can trigger tighter integration of safety and construction process information. Still novel methods and promising results to a realistic field experiment were presented to validate their potential in a practical setting.

What is considered a complex human capability to capture, process, interpret, and assimilate safety information, is yet a challenging task if human-designed systems are to be replicated automatically and in real-time as intelligent prototypes. Improving performance and robustness of tracking site resources for data recording, establishing leading indicator data fusion and inference algorithms, and information sharing through immersive visualization platforms are few of the important examples to improve the current standards-of-practice in workplace safety and health.

Future research has to address the following issues: (a) construction site progress monitoring through fully-automated point cloud to building information model linkage, (b) optimal sensor selection based on technical as well as return-on investment criteria, (c) effective sensor site layout design and implementation in the field, (d) wearable devices that comply with (e) ethical issues involving humans in personal data collection on construction sites, and (f) define and meet the criteria for realistic use and test cases in the harsh construction environment.

\section{REFERENCES}

Bosche, F. and Haas, C.T. (2008). Automated retrieval of 3D CAD model objects in construction range images, Automation in Construction, Elsevier, 17(4), 499-512, http://dx.doi.org/10.1016/j.autcon.2007.09.001.

Golovina, O., Teizer, J. and Pradhananga, N. (2016). Heat Map Generation for Predictive Safety Planning: Preventing Struck-by and Near Miss Interactions between Workerson-Foot and Construction Equipment, Automation in Construction, Elsevier, 71, 99115, http://dx.doi.org/10.1016/j.autcon.2016.03.008.

Hinze, J.W. and Teizer, J. (2011). Visibility-Related Fatalities Related to Construction Equipment, Journal of Safety Science, Elsevier, 49(5), 709-718, http://dx.doi.org/10.1016/j.ssci.2011.01.007. 
Melzner, J, Zhang, S., Teizer, J. and Bargstädt, H.-J. (2013). “A case study on automated safety compliance checking to assist fall protection design and planning in building information models", Construction Management and Economics, Taylor \& Francis, Special Issue on Occupational Health and Safety in the Construction Industry, 31(6), 661-674, http://www.tandfonline.com/doi/abs/10.1080/01446193.2013.780662.

Pradhananga, N. and Teizer, J. (2013). Automatic Spatio-Temporal Analysis of Construction Equipment Operations using GPS Data, Automation in Construction, Elsevier, 29, 107-122, http://dx.doi.org/10.1016/j.autcon.2012.09.004.

Pradhananga, N. (2014). Construction site safety analysis for human-equipment interaction using spatio-temporal data, Doctoral thesis, Georgia Institute of Technology, Atlanta, Georgia, USA, http://hdl.handle.net/1853/52326.

Ray, S.J. and Teizer, J. (2016). Dynamic Blindspots Measurement for Construction Equipment Operators, Safety Science, Elsevier, 85, 139-151, http://dx.doi.org/10.1016/j.ssci.2016.01.011.

Sacks, R., Radosavljevic, M. and Barak, R., (2010). Requirements for building information modeling based lean production management systems for construction, Automation in Construction, 19, 641-655, doi:10.1016/j.autcon.2010.02.010.

Siebert, S. and Teizer, J. (2014). Mobile 3D Mapping for Surveying Earthwork Projects using an Unmanned Aerial Vehicle (UAV) System, Automation in Construction, Elsevier, 41, 1-14, http://dx.doi.org/10.1016/j.autcon.2014.01.004.

Tang, P., Huber, D., Akinci, B., Lipman, R. and Lytle, A. (2010). Automatic reconstruction of as-built building information models from laser-scanned point clouds: A review of related techniques, Automation in Construction, Elsevier, 19(7), 829-843, http://dx.doi.org/10.1016/j.autcon.2010.06.007.

Teizer, J., Allread, B.S. and Mantripragada, U. (2010). Automating the Blind Spot Measurement of Construction Equipment. Automation in Construction, Elsevier, 19(4), 491-501, http://dx.doi.org/10.1016/j.autcon.2009.12.012.

Teizer, J. and Cheng, T. (2015), Proximity Hazard Indicator for Near Miss Location Recording and Mapping of Workers-on-Foot Interactions with Construction Equipment and Geo-Referenced Hazard Areas, Automation in Construction, Elsevier, Vol. 60, pp. 58-73, http://dx.doi.org/10.1016/j.autcon.2015.09.003.

Teizer, J. (2016). Right-time vs. Real-time Pro-active Construction Safety and Health System Architecture, Construction Innovation: Information, Process, Management, Emerald, 16(3), 253-280, http://dx.doi.org/10.1108/CI-10-2015-0049.

Waehrer, G.M., Dong, X.S., Miller, T. Haile, E. and Men, Y. (2007), Costs of occupational injuries in construction in the United States. Accident Analysis \& Prevention, Elsevier, Vol. 39, No. 6, pp. 1258-1266.

Zhang, S., Boukamp, F. and Teizer, J. (2015). Ontology-Based Semantic Modeling of Construction Safety Knowledge: Towards Automated Safety Planning for Job Hazard Analysis (JHA), Automation in Construction, Elsevier, 52, 29-41, http://www.itcon.org/2015/19.

Zhang, G., Vela, P.A., Karasev, P. and Brilakis, I. (2015). A Sparsity-Inducing Optimization-Based Algorithm for Planar Patches Extraction from Noisy Point-Cloud Data, 30(2), 85-102, DOI: 10.1111/mice.12063. 